\title{
Influence of chelation ratio of metal alkoxides on aging of PZT 53/47 sol-gel-based precursors
}

\author{
A SUÁREZ-GÓMEZ*, M SANCHEZ-TIZAPA, R CASTAÑEDA-VALDERRAMA and \\ M A CARREÓN-ÁLVAREZ \\ CUVALLES-UdG, Carr. GDL-Ameca km 45.5, CP 46600 Ameca, Jalisco, Mexico
}

MS received 21 September 2013

\begin{abstract}
The high reactivity of metal alkoxides, common starting reagents in sol-gel-based synthesis routes, generally demands the use of chelating agents in order to avoid fast hydrolysis and also to allow an easier manipulation of intermediates as well as final solutions. Under these conditions, stability issues sometimes can be linked to chelation issues that, therefore, should deserve a proper study suited to the material system of interest and, more specially, when considering the use of sol-gel-based precursors in research, small-scale applications and industry. In this work, we explore the sol-gel-based synthesis route of lead zirconate titanate $\left(\mathrm{Pb}\left(\mathrm{Zr}_{0.53} \mathrm{Ti}_{0.47}\right) \mathrm{O}_{3}\right.$ or PZT 53/47) using acetylacetone-chelated propoxides as intermediate reactants. Our main purpose here is to analyse the influence of the alkoxides:acetylacetone chelation ratio on the time evolution of mean particle size in the resultant organic colloidal dispersion used as PZT precursor. Purposely, we explored three different scenarios for chelation: (i) defective chelation, (ii) optimal chelation and (iii) excessive chelation. The time dependence of mean particle size was recorded by dynamic light scattering (DLS) measurements and aggregation kinetics was then explored by considering a diffusion-limited colloidal aggregations (DLCA) model.
\end{abstract}

Keywords. Sol-gel; dynamic light scattering; PZT; aggregation kinetics; colloidal dispersion; chelating agent.

\section{Introduction}

Synthesis of inorganic crystals by sol-gel-based methods (Brinker and Scherer 1990) is still one of the most successful and widely used chemical routes due to their relatively low cost and high degree of homogeneity when mixing the appropriate organic precursors at a molecular level. The commercial availability of starting reagents typically used in this method makes almost unavoidable the use of highly reactive metal alkoxides that, although allowing the synthesis of ceramic materials at lower temperatures than other common solid state reactions, could easily tend to hydrolyse during manipulation and also during the first stages of the chemical synthesis. Moreover, in cases where gelification turns out to be an undesirable process and shelf life needs to be maximized, post-synthesis hydrolysis is also a key factor to consider.

The significant reactivity of this family of compounds, mainly due to the highly ionic metal-oxygen bond, could be circumvented by using polar organic solvents with appropriate ligands that could stabilize-chelate-the metallic centres. This kind of solvents - chelating agents range from carboxylic acids, i.e. acetic acid $\left(\mathrm{CH}_{3} \mathrm{COOH}\right.$ or $\mathrm{HOAc})$, to diketones, i.e. acetylacetone $\left(\mathrm{CH}_{3} \mathrm{COCH}_{2}-\right.$

*Author for correspondence (amaury.sg@gmail.com)
$\mathrm{COCH}_{3}$ or AcacH), or glycol ethers, i.e. 2-methoxyethanol $\left(\mathrm{CH}_{3} \mathrm{OCH}_{2} \mathrm{CH}_{2} \mathrm{OH}\right.$ or 2-MOE). Taking into account the strong interrelationship among the nature of the reactants and the chelating agents, as well as its influence on several structural properties of the final compound, multiple studies have been reported in this respect for particular synthesis routes, both theoretical and experimental, and many possible chelation reaction pathways have been proposed and dissected. Particularly, the chemistry of metal alkoxides has been intensively studied by Livage et al (1988) and Sedlar and co-worker (1995).

Ferroelectrics, on the other hand, are among the many materials that have been intensively studied, when synthesized by sol-gel based routes (Schwartz and Narayanan 2008; Talapin 2008). Their spreadable technological use, as well as the increasing needs of miniaturization imposed by the electronic industry, have made possible the obtainment of thin ferroelectric films, nanoparticles, nanoceramics and nanostructured composite materials with excellent morphological and operational characteristics for several applications (Goel and Biradar 2013; Prabu et al 2013; Wan et al 2013).

One of the backbones of the ferroelectrics industry is the lead zirconate titanate [PZT: $\mathrm{Pb}\left(\mathrm{Zr}_{1-x} \mathrm{Ti}_{x}\right) \mathrm{O}_{3}$ ] ceramic system, a well known $\mathrm{ABO}_{3}$ perovskite with a wide range of industrial applications since the early 1950s of the 20th century. The sol-gel synthesis of PZT ceramics has 
evolved a lot, since it was first reported in the mid-1980s of the last century; thermal treatments, precursors, additives, diluents and stabilizers have been incorporated and/or modified in order to suit a specific need (Budd et al 1985; Majumder et al 2002; Schneller and Waser 2002).

One of the most important steps to watch over in any PZT alkoxy-based synthesis route is the chelation of the $\mathrm{Zr}$ and $\mathrm{Ti}$ alkoxides (by using acetylacetone, in our case), specially because the resultant chelated metal complexes may be determinant to turn the final solution into a hydrophobic sol, poorly hydrolysable and, therefore, very stable. Stability issues, as expected, tend to be crucial when using sol-gel based precursors in research, smallscale applications and industry (Balma et al 2011; Fuentes-Fernandez et al 2011; Zhu et al 2011).

The time evolution of the average particle size could be easily correlated with solution stability as long as we consider this dependence to be an indicator of several undesirable processes that could be taking place in the sol due to aging or other instabilities, inherently associated to the chemical environment: aggregation, flocculation and sedimentation. Aggregation, eventhough is a reversible process, is a good indicator of instability since the other two processes, which are not reversible, generally follows after some time. Moreover, the average particle size, considered as the final result of the conjugate action of several physicochemical variables, can also be regarded as the definitive experimental variable on which any solgel post processing technique should be based on as it explicitly defines the size range of the so called 'building blocks' for bottom-up studies or applications.

In this work we will analyse the stability of several sol-gel based precursors of PZT 53/47 synthesized with different chelation ratios with acetylacetone. The aging behaviour of the mean particle size as well as some features of the aggregation kinetics will also be shown and discussed.

\section{Sol-gel synthesis and chelation}

The acetic acid, acetylacetone and 2-methoxyethanol propoxy-based sol-gel synthesis route and some of its physicochemical features have been the subject of some of our earlier reports so a very detailed description of synthesis process can be found elsewhere (Suárez-Gómez et al 2008).

Starting reagents were: (1) lead (II) acetate trihydrate $\left(\mathrm{Pb}(\mathrm{OAc})_{2} \cdot 3 \mathrm{H}_{2} \mathrm{O}\right.$, Aldrich, 99.99\% pure), (2) acetic acid (HOAc, Sigma-Aldrich, 99.7\%), (3) acetylacetone (AcacH, Sigma-Aldrich, 99\%), (4) 2-methoxyethanol anhydrous (2-MOE, Sigma-Aldrich, 99.8\%), (5) zirconium(IV) propoxide $\left(\mathrm{Zr}(\mathrm{OPr})_{4}\right.$, Aldrich, $70 \mathrm{wt} \%$ in 1-propanol), and (6) titanium(IV) propoxide $\left(\mathrm{Ti}\left(\mathrm{O}^{\mathrm{i}} \mathrm{Pr}\right)_{4}\right.$, Aldrich, 98\%).
After dissolving lead acetate in acetic acid with a 1:3 molar ratio (solution A), stoichiometric amounts of zirconium and titanium propoxides were mixed with acetylacetone (solution $\mathrm{B}$ ) with a $1: R$ molar ratio. According to our experimental conditions, different values of $R$ could lead us to three different chemical scenarios for solution B (Sedlar and Sayer 1995):

$$
\begin{aligned}
& 0 \cdot 53 \mathrm{Zr}\left(\mathrm{O}^{\mathrm{i}} \mathrm{Pr}\right)_{4}+0 \cdot 47 \mathrm{Ti}\left(\mathrm{O}^{\mathrm{i}} \mathrm{Pr}\right)_{4} \\
& +R \cdot \text { AcacH } \stackrel{T \sim 90^{\circ} \mathrm{C}}{\underset{t \sim 4 \mathrm{~h}}{\longrightarrow} \cdots} \\
& +(R-\alpha) \operatorname{Ti}(\text { Acac })_{2}\left(\mathrm{O}^{\mathrm{i}} \mathrm{Pr}\right)_{2}+\cdots \\
& +(0.53-\alpha) \operatorname{Zr}\left(\mathrm{O}^{\mathrm{i}} \mathrm{Pr}\right)_{4}+(0.47-R+\alpha) \operatorname{Ti}\left(\mathrm{O}^{\mathrm{i}} \mathrm{Pr}\right)_{4} \text {, }
\end{aligned}
$$

(ii) if $R=3 \cdot 06: 3 \cdot 06 \mathrm{HO}^{\mathrm{i}} \mathrm{Pr}+0 \cdot 53 \mathrm{Zr}(\text { Acac })_{4}$

$$
+0 \cdot 47 \mathrm{Ti}(\text { Acac })_{2}\left(\mathrm{O}^{\mathrm{i}} \mathrm{Pr}\right)_{2} \text {, }
$$

(iii) if $R>3.06: 3 \cdot 06 \mathrm{HO}^{\mathrm{i}} \mathrm{Pr}+0 \cdot 53 \mathrm{Zr}(\text { Acac })_{4}$

$$
+0 \cdot 47 \mathrm{Ti}(\text { Aсас })_{2}\left(\mathrm{O}^{\mathrm{i}} \mathrm{Pr}\right)_{2}+(R-3 \cdot 06) \mathrm{AcacH} .
$$

As can be seen, we have denoted by $\alpha$, the amount of $\mathrm{Zr}$ propoxide that reacts with acetylacetone in the case, where $R$ is smaller than certain 'optimal' value $R_{\text {optimal }}=$ 3.06 for PZT 53/47. This value, easily extrapolated for any $(1-x) / x \mathrm{Zr} / \mathrm{Ti}$ molar ratio could be obtained according to the reaction:

$$
\begin{aligned}
& (1-x) \operatorname{Zr}\left(\mathrm{O}^{\mathrm{i}} \mathrm{Pr}\right)_{4}+x \operatorname{Ti}\left(\mathrm{O}^{\mathrm{i}} \mathrm{Pr}\right)_{4} \\
& +2(2-x) \operatorname{AcacH} \frac{T \sim 90^{\circ} \mathrm{C}}{t \sim 4 \mathrm{~h}} 2(2-x) \mathrm{HO}^{\mathrm{i}} \mathrm{Pr}+\cdots \\
& +(1-x) \operatorname{Zr}(\text { Acac })_{4}+x \operatorname{Ti}(\text { Acac })_{2}\left(\mathrm{O}^{\mathrm{i}} \mathrm{Pr}\right)_{2} .
\end{aligned}
$$

Apparently, choosing optimal chelation ratios not only guarantees complete chelation of the alkoxides, but also minimizes the amount and variety of chemical species in solution. Consequently, different $R$ values were chosen for the purposes of this study: $R=2.50,2 \cdot 75$ (defective chelation), 3.06 (optimal chelation) and 3.25 (excessive chelation).

Solutions A and B were then mixed with 2-MOE to attain a concentration value of $0.35 \mathrm{M}$ for PZT 53/47 in the precursor solution. After stirring for about $10 \mathrm{~h}$, approximately $1.5 \mathrm{~mL}$ of as-synthesized sols were stored in polystyrene cells and particle size measurements were carried out by means of dynamic light scattering (DLS) technique in a Zetasizer Nano ZS90 (Malvern Instruments Ltd.). For this purpose, several solvent parameters were needed for further processing of dispersed light intensity, namely viscosity $\left(\eta_{2 \mathrm{MOE}} \sim 1.5410 \mathrm{cP}\right)$, dielectric constant $\left(\varepsilon_{2-\mathrm{MOE}} \sim 16 \cdot 9\right)$ and refraction index 


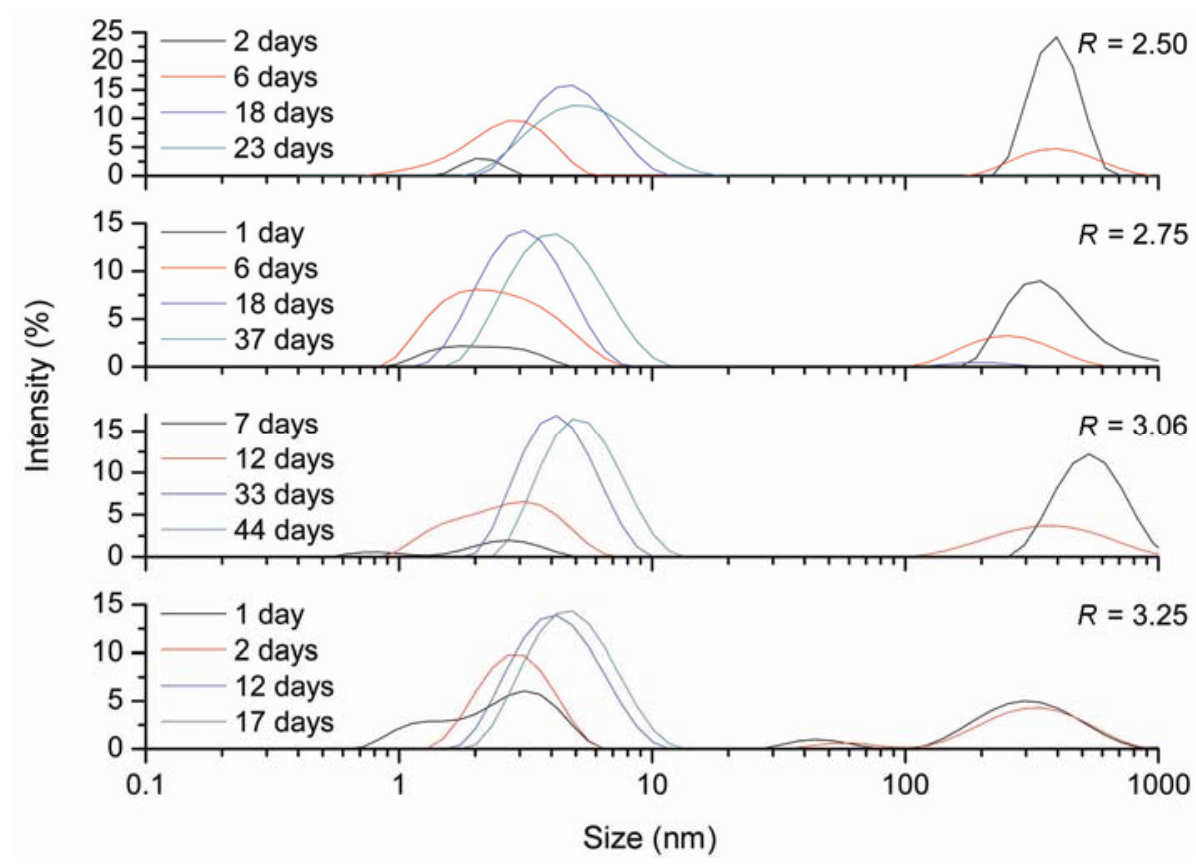

Figure 1. 'As-synthesized' and 'aged' distribution curves recorded by DLS measurements for the samples under study.

$\left(n_{2-\mathrm{MOE}} \sim 1 \cdot 33\right)$ (Das and Hazra 1998). Size distribution curves were recorded for different aging times, ranging from early days after synthesis up to until 1.5 months, when possible.

\section{Results and discussion}

Figure 1 shows, for each $R$-chelated precursor, four of the measured size distribution curves by DLS, considering two distinctive stages: samples aged just a few days after synthesis and samples considered to be 'old enough'.

As can be seen, as-prepared sols featured multimodal distributions with a noticeable percentage of particles that could even have sizes sometimes above $1 \mu \mathrm{m}$. As solutions are aged, these distributions tend to be unimodal featuring a mean particle size well below $10 \mathrm{~nm}$. As stated in an earlier work (Suárez-Gómez et al 2010), from here it is evident that there is no rule of thumb related to the need of using fresh, as-prepared solutions for synthesizing this kind of material at any desirable working scale. Furthermore, aged samples could be more suitable not only for bulk materials, but also for thin films or nanostructures synthesis due to the better homogeneity regarding particle size distribution in all the cases under study.

This behaviour can be explained, in principle, on the basis of the chemical reaction that was described earlier for obtaining what we called solution $\mathrm{B}$ by making the plausible assumption that solution $\mathrm{B}$ is a dispersion containing the $\mathrm{Ti}(\mathrm{Acac})_{2}(\mathrm{OPr})_{2}$ compound at molecular level and submicrometric aggregates of $\operatorname{Zr}(\text { Acac })_{4}$ that are redissolved afterwards when mixed with the acetate rich solution A and 2-MOE (Suárez-Gómez et al 2008, 2010; Stawski et al 2012).

As a consequence, time evolution of PZT precursors under study should be roughly divided into at least two stages when dealing with aggregation kinetics. For this purpose, we chose to consider two fairly accurate descriptors of an almost unimodal colloidal dispersion: (a) population percentage of the leftmost distribution peak must be consistently greater than 85\% and (b) polydispersity index, as reported by the DLS equipment, must be consistently smaller than $33 \%$.

The simultaneous verification of (a) and (b) should give us, in our opinion, a fair estimate of when to consider that samples have aged enough to be considered unimodal and a practical merit figure, that will be referred as 'unimodal age' from now on, for any colloidal dispersion featuring a multimodal/unimodal transformation when aged. Figure 2 shows behaviour of the aforementioned parameters with the aging time as well as dashed circles used to highlight the 'unimodal age' of each sample.

Figure 3 shows, on the other hand, three size distribution curves for every $R$-chelated sample by considering their respective 'unimodal age' and also comparing with the previous and the next measurements of each set. As depicted, in the vicinity of the 'unimodal age' skewed distribution curves transform into bell-shaped ones, somehow confirming the validity of our considerations based on polydispersity and peak population. 
At this stage, even if it is not plausible to consider our samples to be monodisperse, we could assert that precursor solutions are, at least, sufficiently homogeneous as to allow a better atomic assembly for any post-synthesis processing as required, for instance, in electrophoretic deposition (EPD), spin-coating deposition (SCD) or just a common calcination and sintering route for bulk nanoceramics fabrication.

From the results shown above, it is worth to note that the dependence of the 'unimodal age' with the degree of chelation $(R)$. As can be seen in figure 2 and in table 1 , as $R$ increases, samples need more time to homogenize due to higher stabilization of the alkoxides reactants via chelation and this also implies longer times for the appropriate redissolution of the resultant $\operatorname{Zr}(\text { Acac })_{4}$ complexes. Significantly, for $R$ values greater than the
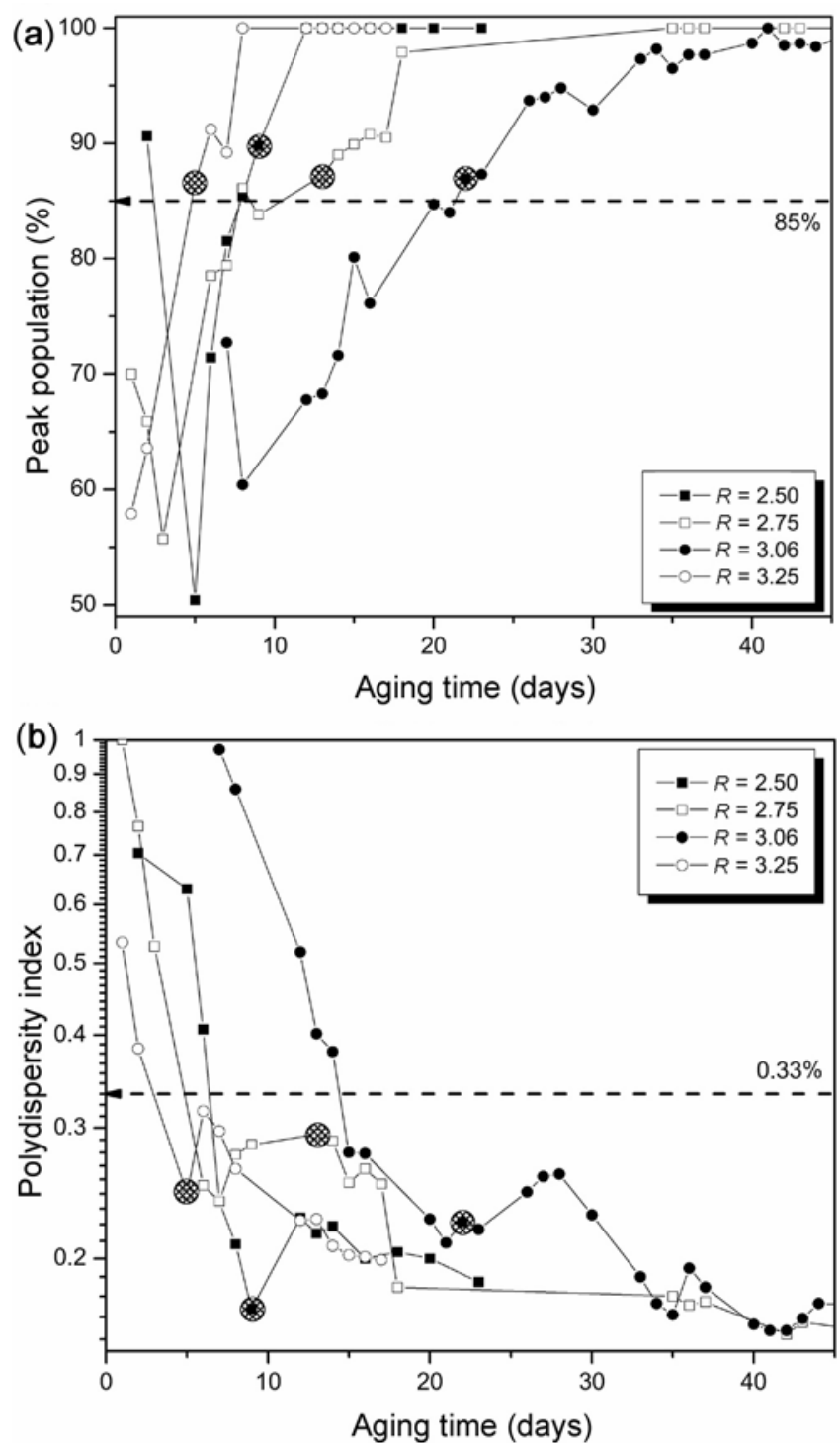

Figure 2. (a) Peak population and (b) polydispersity index for our samples as non-direct indicators of unimodality; 'unimodal age' is highlighted on each case. optimal chelation ratio, $R_{\text {optimal }}=3 \cdot 06$, the 'unimodal age' drastically drops to a value, i.e. even smaller than the reported for the less-chelated sample under study. This behaviour can be explained by taking into account that, for $R>R_{\text {optimal }}$, the amount of remnant AcacH-a weak acid-will imply a more acidic medium (see $\mathrm{pH}$ values in table 1) which is a favourable ionic environment to achieve shorter times for the redissolution of $\operatorname{Zr}(\text { Acac })_{4}$ complexes.

Time evolution of mean particle size for the most prominent distribution peak of every $R$-chelated sample under study is depicted in figure 4, where arrows indicate the 'unimodal age' on each case. As a general tendency, precursor particles are well below the $10 \mathrm{~nm}$ range, which is an interesting feature with an immediate practical impact.

In order to explore the significance of the increasing trend that can be seen in the figure, we first carried out Kolmogorov-Smirnov (Kolmogorov 1933; Smirnov 1948) and Shapiro-Wilk (Shapiro and Wilk 1965) normality tests that were followed by a statistical analysis based on two-tailed paired and unpaired $t$-tests to our datasets. The results of both tests, as well as their corresponding $p$-values are shown in table 2 .

The shaded rows highlight the results, where $p$ value $>0.05$, implicating that, according to our recorded experimental datasets, samples with $R=2.50$ and $R=3.25$ could be treated as most statistically equivalent group (Group 1) and the same can be said about samples with $R=3.06$ and $R=2.75$ (Group 2), also highlighted in table 2. Consequently, we will also discuss the aging behaviour of our samples taking into account this fact.

At this moment, we can not give a proper explanation for this behaviour but it must be observed that the samples in Group 1 are both the less $(R=2.50)$ and the more $(R=3 \cdot 25)$ chelated samples of our study. Eventhough

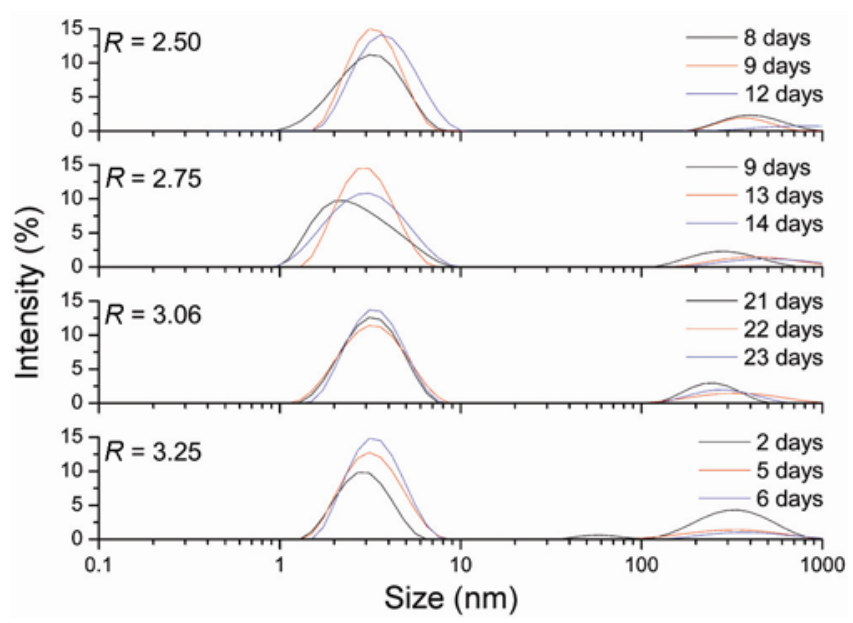

Figure 3. Size distribution curves when going through 'unimodal age'; changes in peaks shape and population are clearly seen. 
Table 1. Summary of some relevant parameters discussed above and, for the purposes of our study, associated to solution stability and aging behaviour.

\begin{tabular}{lcccccc}
\hline $\begin{array}{l}\text { Chelation } \\
\text { ratio }(R)\end{array}$ & $\begin{array}{c}\text { Unimodal } \\
\text { age (days) }\end{array}$ & $\begin{array}{c}\text { Sol. } \mathrm{pH} \\
\text { (as synthesized) }\end{array}$ & $\begin{array}{c}\text { Sol. } \mathrm{pH} \\
\text { (unimodal age) }\end{array}$ & $\begin{array}{c}\left\langle d_{\mathrm{f}}\right\rangle_{\mathrm{before}} \\
(\mathrm{nm})\end{array}$ & $\begin{array}{c}\left\langle d_{\mathrm{f}}\right\rangle_{\mathrm{after}} \\
(\mathrm{nm})\end{array}$ & $\begin{array}{c}\text { Shelf life } \\
\text { (days) }\end{array}$ \\
\hline 2.50 & 9 & 5.08 & 4.99 & 0.682 & 1.062 & $\sim 25$ \\
2.75 & 13 & 5.55 & 4.94 & 0.993 & 1.484 & $\sim 55$ \\
3.06 & 22 & 5.10 & 5.34 & 0.726 & 0.647 & $\sim 120$ \\
3.25 & 5 & 4.71 & 4.73 & Not determined & 0.859 & $\sim 20$ \\
\hline
\end{tabular}

Table 2. Summary of the paired and unpaired $t$-tests carried out to our whole datasets. Statistically equivalent groups ( $p$-value $>0 \cdot 05)$ are highlighted.

\begin{tabular}{lrrrrc}
\hline & \multicolumn{2}{c}{ Unpaired samples } & & \multicolumn{2}{c}{ Paired samples } \\
\cline { 2 - 3 } \cline { 5 - 6 } & $|t(24)|$ & $p$-value & & $|t(24)|$ & $p$-value \\
\hline$R 250-R 275$ & 3.795 & 0.001 & & 6.229 & $<0.001$ \\
$R 250-R 306$ & 4.192 & $<0.001$ & & 5.913 & $<0.001$ \\
$R 250-R 325$ & -0.730 & 0.469 & & -2.819 & 0.10 \\
$R 275-R 306$ & 0.707 & 0.483 & & 2.630 & 0.15 \\
$R 275-R 325$ & -6.297 & $<0.001$ & & -14.129 & $<0.001$ \\
$R 306-R 325$ & -7.007 & $<0.001$ & & -12.086 & $<0.001$ \\
\hline
\end{tabular}

the physicochemical environment is not the same from one sample to the other, excessive chelation and defective chelation could lead, statistically speaking, to a similar regime of aggregation kinetics. The existence of a hypothetical 'threshold chelation rate' between statistically equivalent populations is something we are not able to discuss right now and will deserve future hindsights.

\subsection{R-chelated samples treated as different chemical species}

As depicted in figure 4, non-linear least squares fitting (NLLSF) was carried out before and after the 'unimodal age' based on a diffusion-limited colloid aggregation (DLCA) regime of aggregation kinetics (Lin et al 1990a) and described by the relationship:

$$
\langle d\rangle=d_{0}+A t^{1 /\left\langle 1 / d_{\mathrm{f}}\right\rangle},
$$

where the parameters $A, d_{0}$ and $\left\langle d_{\mathrm{f}}\right\rangle$ were fitted to the available experimental data. According to our chemical route and experimental procedure, a reaction-limited colloid aggregation (RLCA) (Lin et al 1990b) scenario was discarded because any reaction-limited process should occur within a few hours after synthesis and that is why samples were stirred for almost $10 \mathrm{~h}$ afterwards. Resultant methoxyethoxy-acetate compounds, a feasible product for the case of $\mathrm{Pb}$ rich solution $\mathrm{A}$ (Kolb et al 1997), tend to form very quickly as well as any further 2MOE chelation of the remnant unreacted alkoxides when $R<R_{\text {optimal }}$. It must be noted, however, that these supposedly RLCA-dominated first stages of sol formation could also be considered for a future investigation.

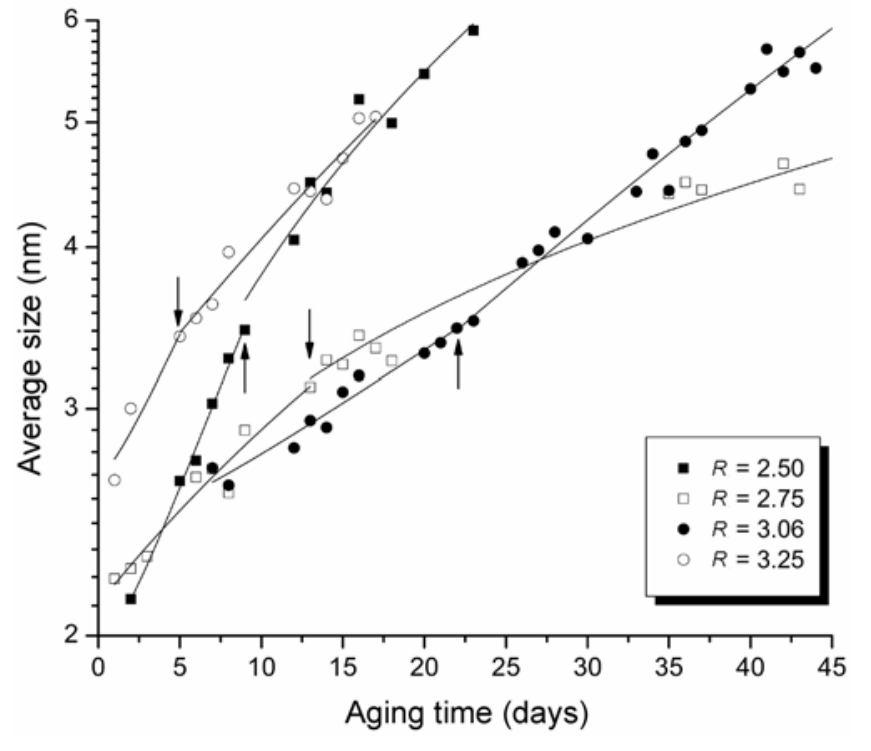

Figure 4. Time evolution of average particle size $\langle d\rangle$ for the specimens under study and treated as different chemical entities. NLLSF curves based on a DLCA model are also shown.

The more relevant fitting parameter, $\left\langle d_{\mathrm{f}}\right\rangle$, corresponds to the average fractal dimension of the colloidal aggregates and its values are shown in table 1 . It should be considered that $\left\langle d_{\mathrm{f}}\right\rangle$ values were calculated by taking into account only the average size measured for the leftmost distribution curve and before the 'unimodal age', the coexistence of two or more populations have been neglected. This fact could lead us to reconsider the physical 
meaning of the calculated value for $\left\langle d_{f}\right\rangle,\left\langle d_{f}\right\rangle_{\text {before }}$ in table 1 , as exclusively pertaining to a single size interval and not to the whole colloidal dispersion. On the other hand, and as can be seen in figure 4 , only three measurements were recorded for the sample with $R=3.25$ before 'unimodal age', thus making impossible a proper NLLSF based on the power law given by (3); on its place, a linear fit was carried out.

According to our results, the average fractal dimension of our samples increases after reaching its 'unimodal age' except for the optimally chelated solution $\left(R_{\text {optimal }}=3 \cdot 06\right)$, where $\left\langle d_{\mathrm{f}}\right\rangle$ slightly decreases. Consequently, slope changes at 'unimodal age' between the two curves are noticeable for every $R$-chelated sample, even though, for optimal chelation, it behaves more like a single continuous line. These results, in our opinion, highlight the importance of an optimal full chelation when analysing the aggregation kinetics in this kind of sols as it could be statistically treated with a single average value for the fractal dimension right from the final step of the synthesis and, therefore, providing a more predictable aging behaviour that could be exploited in any industry where a fine-tuned precursor particle size is a technological demand (Talapin 2008).

It is worth to note that the less chelated sample has a very distinguishable discontinuity at 'unimodal age', maybe suggesting that the kinetics of the aggregation process should be more appropriately described by an effective aggregation model considering a mixture of average fractal dimensions (Gharagozloo and Goodson 2010). This assumption relies heavily on the feasibility of the formation of 2-MOE chelated complexes when mixing 2-MOE with the unreacted metal alkoxides of solution B (Livage et al 1988). As can be seen, for $R=2 \cdot 75$, discontinuity at the 'unimodal age' persists but slighter. Afterwards, when optimal full chelation is achieved, this discontinuity is neglectable.

Another experimental result that deserves to be mentioned is that the smaller $\left\langle d_{\mathrm{f}}\right\rangle$ values attained for completely chelated samples. This fact may lead us to consider that fractal aggregates in these solutions tend to behave like Brownian particles with a small probability for gelification as time, theoretically, tends to infinity (Cecconi et al 2007). Considering this, the relevance of an optimal chelation ratio is again highlighted.

\subsection{R-chelated samples treated as two statistically equivalent groups}

As was explained above, samples were also divided into two groups almost statistically equivalent and, just as was done in §3.1, a non-linear least squares fitting (NLLSF) was carried out based on a diffusion-limited colloid aggregation (DLCA) before and after the 'unimodal age' and the results are shown in figure 5 and in table 3 . It is important to point out that, for this approach, the 'unimodal age' of a group was simply chosen as the biggest 'unimodal age' of the corresponding samples.

In this case, except $\left\langle d_{\mathrm{f}}\right\rangle_{\text {before }}$ for group 2, the fractal dimension attains intermediate values with respect to those previously reported in $\$ 3.1$ and, when considering the structure of our two new datasets, this could be an expected result. On the other hand, discontinuities at 'unimodal age' are still present but somehow slighter than before due to the statistical criterion used to perform this analysis. However, this reinforces what was said earlier about the plausibility of an effective aggregation model considering a mixture of average fractal dimensions.

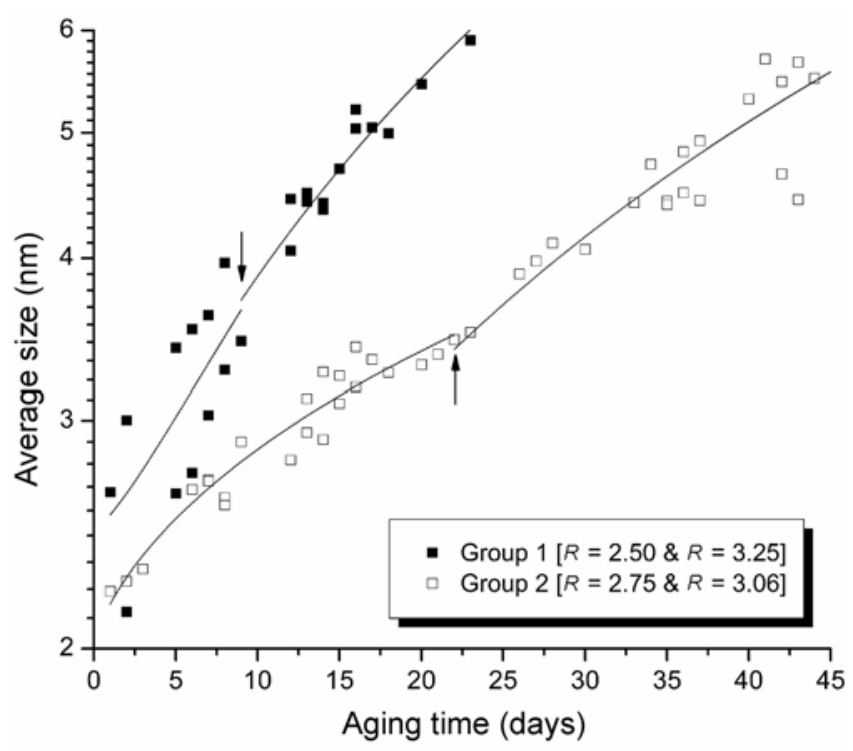

Figure 5. Time evolution of average particle size $\langle d\rangle$ for the specimens under study and treated as two statistically equivalent groups. NLLSF curves based on a DLCA model are also shown.

Table 3. Fractal dimensions before and after 'unimodal age' obtained by a DLCA model fitting by NLLSF to our datasets considering two statistically equivalent groups.

\begin{tabular}{lccc}
\hline Groups & $R$-chelated samples & $\left\langle d_{\mathrm{f}}\right\rangle_{\text {before }}(\mathrm{nm})$ & $\left\langle d_{\mathrm{f}}\right\rangle_{\text {after }}(\mathrm{nm})$ \\
\hline Group 1 & $R=2.50$ & 0.766 & 0.961 \\
& $R=3.25$ & & \\
Group 2 & $R=2.75$ & 1.404 & 0.885 \\
& $R=3.06$ & & \\
\hline
\end{tabular}


Finally, as samples aged, the unavoidable evaporation of solvents and other volatile byproducts turned the assynthesized colloidal dispersions into blurred, thicker and reddish solutions that became immeasurable for the DLS apparatus. Considering this experimental threshold as a good measure of the unsuitability for any immediate technological demand, we used it to roughly estimate the 'shelf life' of our precursors and those values are also shown in table 1 . As reported, optimally chelated sample adds to its previously discussed advantages, a longer stability with shelving time as it lasted several months without any crucial degradation.

\section{Conclusions}

In this paper, and by means of the DLS experimental technique, we analysed the influence of the chelation ratio of metal alkoxides in the aggregation kinetics of solgel based precursors for the synthesis of the technologically important PZT 53/47 material. We also introduced the 'unimodal age' parameter as a merit figure for determining the best conditions under which our resultant colloidal dispersions should be applied, as desired, in any nanometric or submicron working scale. To the best of our knowledge, there are no reports using this kind of approach for studying the stability and aggregation kinetics of PZT sol-gel-based precursors.

It was shown that, for three different possible chemical scenarios, the degree of chelation plays an important role in determining the 'unimodal age', the behaviour of the average fractal dimension for the colloidal aggregates and also the shelf life of our precursors. An optimal chelation ratio for PZT 53/47, $R_{\text {optimal }}=3 \cdot 06$, showed a longer stability and a more predictable aging behaviour as well as a simpler chemical environment due to the minimization of the amount of byproducts in the intermediate steps of the synthesis. These features are currently motivating future works concerning the statistical modelling of such a colloid and also about the extendibility of these conclusions to any similar PZT $(1-x) / x$ precursor.

Besides, it was also shown that, while always working beyond the 'unimodal age', it does not matter what chelation ratio is used; particle size distributions are all unimodal with an average well below $10 \mathrm{~nm}$. Shelf life, however, will always be an issue that must be considered when working with non-optimally chelated metal alkoxides.

\section{Acknowledgements}

Authors would like to acknowledge the funding provided by PIFI (Programa Integral de Fortalecimiento Institucional) to the Project No. P/PIFI-2011-14MSU0010Z16 for the acquisition of the Malvern Zetasizer Nano ZS90 equipment used in this research. Special thanks to Dr O Dalmau-Cedeño (CIMAT, U-Monterrey, Mexico) for his willing collaboration with the authors on the statistical analysis. Students I G Romero-Flores and O ArjónZamorano, financially supported by COECYTJAL Project No. 5-2010-1-937, also collaborated in the experimental part of this work.

\section{References}

Balma D, Lamberti A, Marasso S L, Perrone D, Quaglio M, Canavese G, Bianco S and Cocuzza M 2011 Microelectron. Eng. 882208

Brinker C J and Scherer G W 1990 Sol-gel science: The physics and chemistry of sol-gel processing (San Diego, CA: Academic Press Inc.)

Budd K D, Dey S K and Payne D A 1985 Brit. Ceram Proc. 36 107

Cecconi F, Gonnella G and Saracco G P 2007 Phys. Rev. E: Stat. Nonlin. Soft Matter Phys. 75031111

Das B and D K Hazra 1998 J. Sol. Chem. 271021

Fuentes-Fernandez E, Baldenegro-Perez L, Quevedo-Lopez M, Gnade B, Hande A, Shah P and Alshareef H N 2011 SolidState Electron. 6389

Gharagozloo P E and Goodson K E 2010 J. Appl. Phys. 108 074309

Goel P and Biradar A M 2013 J. Phys. Chem. Solids 74854

Kolb U, Gutwerk D, Beudert R and Bertagnolli H 1997 J. NonCryst. Solids 217162

Kolmogorov A N 1933 G. Ist. Ital. Attuari 483

Lin M, Lindsay H, Weitz D, Ball R, Klein R and Meakin P 1990a Phys. Rev. A: At. Mol. Opt. Phys. 412005

Lin M Y, Lindsay H M, Weitz D A, Klein R, Ball R C and Meakin P 1990b J. Phys. Condens. Matter 23093

Livage J, Henry M and Sanchez C 1988 Prog. Solid State Chem. 18259

Majumder S B, Bhaskar S and Katiyar R S 2002 Integr. Ferroelectr. $\mathbf{4 2} 245$

Prabu M, Shameem Banu I B, Gobalakrishnan S and Chavali M 2013 J. Alloys Compd. 551200

Schneller T and Waser R 2002 Ferroelectrics 267293

Schwartz R W and Narayanan M 2008 Chemical solution deposition-Basic principles In Solution processing of inorganic materials (ed.) D B Mitzi (Hoboken, NJ, USA: John Wiley \& Sons Inc.) p 33

Sedlar M and Sayer M 1995 J. Sol-Gel Sci. Technol. 527

Shapiro S S and Wilk M B 1965 Biometrika 52591

Smirnov N V 1948 Ann. Math. Stat. 19279

Stawski T M, Besselink R, Veldhuis S A, Castricum H L, Blank D H and ten Elshof J E 2012 J. Coll. Interf. Sci. 369184

Suárez-Gómez A, Saniger-Blesa J M and Calderón-Piñar F 2010 Mater. Chem. Phys. 123304

Suárez-Gómez A, Sato-Berrú R, Toscano R A, Saniger-Blesa J M and Calderón-Piñar F 2008 J. Alloys Compd. 450380

Talapin D V 2008 Engineered nanomaterials as soluble precursors for inorganic films In Solution processing of inorganic materials (ed.) D B Mitzi (Hoboken, NJ, USA: John Wiley \& Sons Inc.) p 313

Wan Q, Gu Q, Xing J and Chen J 2013 Mater. Lett. 9252

Zhu Y, Liu W, Jia K, Liao W and Xie H 2011 Sensor Actuators A167 495 